\title{
Building evidence to guide PrEP introduction for adolescent girls and young women
}

\author{
Nanlesta Pilgrim \\ Population Council \\ Sanyukta Mathur \\ Population Council \\ Ann Gottert \\ Population Council \\ Naomi Rutenberg \\ Population Council \\ Julie Pulerwitz \\ Population Council
}

Follow this and additional works at: https://knowledgecommons.popcouncil.org/departments_sbsr-hiv

Part of the Demography, Population, and Ecology Commons, Family, Life Course, and Society Commons, International Public Health Commons, and the Maternal and Child Health Commons How does access to this work benefit you? Let us know!

\section{Recommended Citation}

Pilgrim, Nanlesta, Sanyukta Mathur, Ann Gottert, Naomi Rutenberg, and Julie Pulerwitz. 2016. "Building evidence to guide PrEP introduction for adolescent girls and young women." Washington, DC: Population Council. 


\section{BUILDING EVIDENCE TO \\ GUIDE PREP INTRODUCTION \\ FOR ADOLESCENT GIRLS AND \\ YOUNG WOMEN}

Nanlesta Pilgrim

Sanyukta Mathur, Ann Gottert

Naomi Rutenberg Julie Pulerwitz

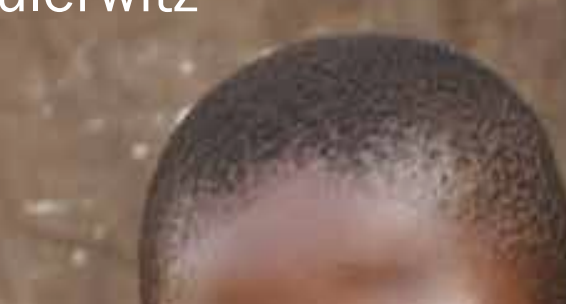




\section{POPULATION \\ COUNCIL}

Ideas. Evidence. Impact.

The Population Council confronts critical health and development issues-from stopping the spread of HIV to improving reproductive health and ensuring that young people lead full and productive lives. Through biomedical, social science and public health research in about 50 countries, the Council works with our partners to deliver solutions that lead to more effective policies, programs, and technologies to improve lives worldwide. Established in 1952 and headquartered in New York, the Council is a nongovernmental, nonprofit organization with an international board of trustees.

Population Council

4301 Connecticut Ave, NW, Suite 280

Washington, DC 20008

USA

Tel: 202-237-9423

Fax: $202-237-8410$

email: publications@popcouncil.org

popcouncil.org

Cover photo: @ Richard Lord, courtesy of the Population Council

Suggested citation: Pilgrim, Nanlesta, Sanyukta Mathur, Ann Gottert, Naomi Rutenberg, and Julie Pulerwitz. 2016. "Building evidence to guide PrEP introduction for adolescent girls and young women." Washington, DC: Population Council.

Any part of this publication may be photocopied without permission from the publisher provided that copies are distributed without charge and that full source citation is provided. The Population Council would appreciate receiving a copy of any materials in which the text is used.

(C) 2016 The Population Council, Inc. 


\section{Building Evidence to Guide PrEP Introduction for Adolescent Girls and Young Women}

Nanlesta Pilgrim, Sanyukta Mathur, Ann Gottert, Naomi Rutenberg, and Julie Pulerwitz 


\section{Acknowledgments}

The authors would like to thank the following people for their technical review and editorial support in producing this document: Gina Dallabetta and Lut Van Damme of the Bill \& Melinda Gates Foundation, and Martha Brady, Barbara Mensch, Barbara Friedland, Miriam Temin, Erin Kiernon, Ellen Weiss, Nrupa Jani, and Sherry Hutchinson of the Population Council. Special thanks to the Bill \& Melinda Gates Foundation for supporting this work. 


\section{Table of Contents}

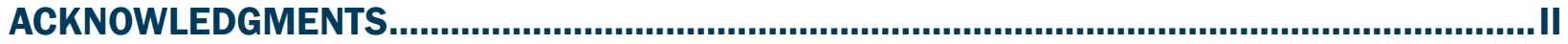

LIST OF ABBREVIATIONS...............................................................................................

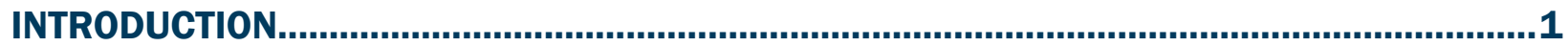

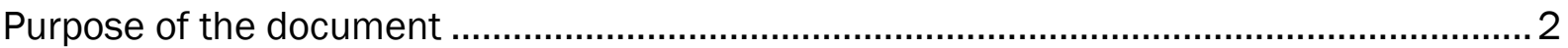

Ethical considerations ...............................................................................................

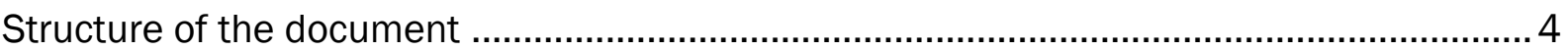

SECTION 1: DEVELOPING A PREP INTRODUCTION STRATEGY FOR AGYW:

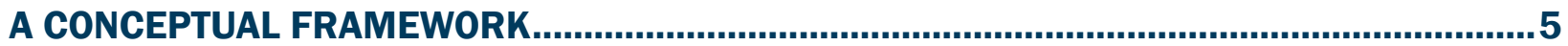

SECTION 2: ENGAGING KEY ACTORS: KEY DOMAINS, METHODS, AND QUESTIONS ............8

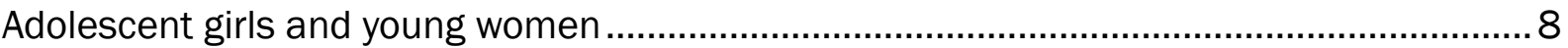

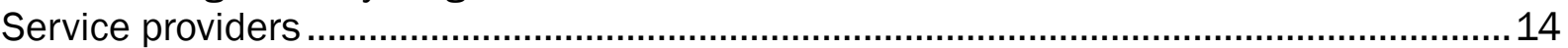

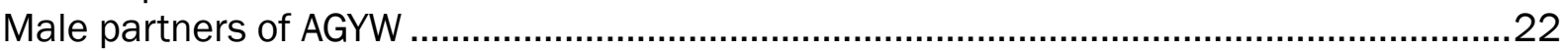

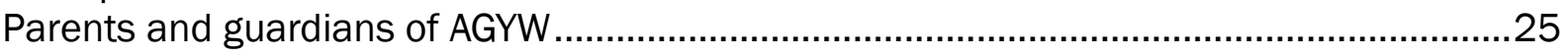

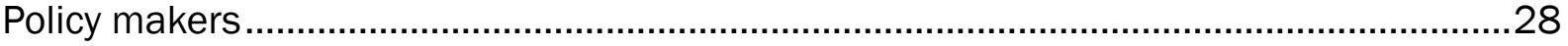

SECTION 3: ADDITIONAL DATA COLLECTION METHODS …................................................ 29

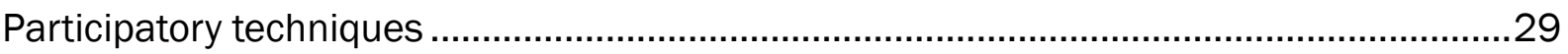

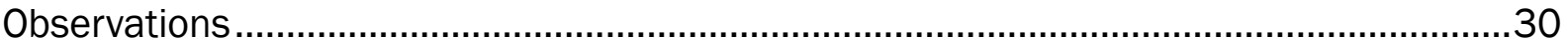

Mystery client method ……………….............................................................................

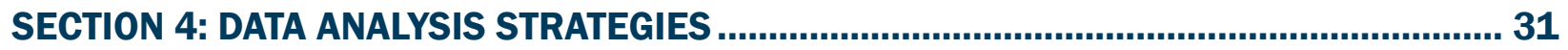

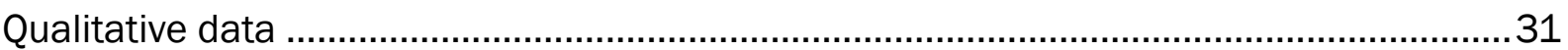

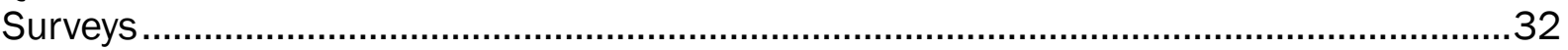

SECTION 5: RESEARCH UTILIZATION ................................................................................ 33

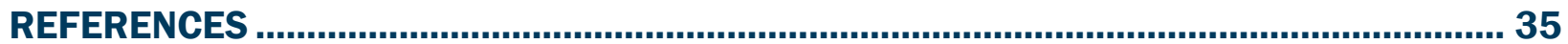




\section{List of Abbreviations}

$\begin{array}{ll}\text { AGYW } & \text { Adolescent girls and young women } \\ \text { ARV } & \text { Antiretroviral } \\ \text { CBO } & \text { Community-based organization } \\ \text { DREAMS } & \text { Determined, Resilient, Empowered AIDS-free, Mentored, and Safe } \\ \text { FGD } & \text { Focus group discussion } \\ \text { FSW } & \text { Female sex worker } \\ \text { IDI } & \text { In-depth interview } \\ \text { NGO } & \text { Nongovernmental organizaton } \\ \text { PEPFAR } & \text { U.S. President's Emergency Plan for AIDS Relief } \\ \text { PrEP } & \text { Pre-exposure prophylaxis } \\ \text { SOAR } & \text { Supporting Operational AIDS Research } \\ \text { UNAIDS } & \text { Joint United Nations Programme on HIV/AIDS } \\ \text { WHO } & \text { World Health Organization }\end{array}$




\section{Introduction}

Adolescent girls and young women (AGYW), ages 15-24 years, account for 75 percent of young people in sub-Saharan Africa who are living with HIV. ${ }^{1}$ As many as 7,000 new infections a week are occurring among the most vulnerable AGYW in Eastern and Southern Africa; AIDS is the leading cause of death for adolescent girls ages 10-19 in Africa. ${ }^{1}$ In sub-Saharan Africa, women acquire HIV five to seven years earlier than men, and young women are at an increased risk of HIV acquisition compared to young men. ${ }^{1}$ To date, prevention efforts have not had the maximum impact on AGYW's risk of acquiring HIV. ${ }^{2}$ The Joint United Nations Programme on HIV/AIDS (UNAIDS), highlights gender-based violence, limited access to health services, limited education, and policies or systems that exclude young women as the key contributors to AGYW's increased vulnerability to HIV. ${ }^{1}$ The introduction of pre-exposure prophylaxis (PrEP) within the context of a comprehensive HIV prevention package, would make a new HIV prevention option available for AGYW, offering them an opportunity for more control of their sexual health.

Oral PrEP, the use of antiretroviral (ARV) medications by HIV-negative persons to prevent the acquisition of HIV, is a new biomedical intervention that has the potential to substantially reduce HIV acquisition among those most vulnerable to HIV. Recent evidence indicates that PrEP is efficacious in different populations at high risk of HIV acquisition-in studies with high adherence, HIV infection risk was reduced by 70 percent. $^{3}$ Trial results have shown that oral PrEP in men who have sex with men significantly reduces the risk of HIV acquisition by as much as 92 percent when taken as prescribed. ${ }^{4}$ Results on the efficacy of PrEP for women, aged 18 and older, are
Keeping adolescent girls and young women HIV- and AIDS-free is critical for their well-being and health, the health of their families and communities, and their countries' future.

mixed, most likely associated with different levels of adherence. ${ }^{3}$ It also appears that women need to take daily oral PrEP for a longer period of time than men before they acquire protective levels of the drug in their body. ${ }^{2,5-7}$ For young women, emerging data show that when women know that PrEP is effective, they use it more consistently. ${ }^{8}$ Given their increased vulnerability to HIV infection, PrEP could have a significant impact on HIV acquisition among AGYW, if they are able to effectively access and use PrEP. Thus, we need to fill existing gaps in knowledge about how different stakeholders will perceive PrEP, how AGYW will use it in real-world settings, and how to design and implement effective service delivery models.

Currently there is great interest and momentum in bringing PrEP to populations at substantial risk of HIV, including AGYW. Decreasing infections among young people is a key aspect of the global ALL IN intitiave to accelerate the fight against AIDS for adolescents. Further, the World Health Organization $(\mathrm{WHO})^{4}$ recommends the availability and use of oral PrEP to mitigate HIV acquisition. The DREAMS (Determined, Resilient, Empow- 
Countries will need to develop a vision for how PrEP for AGYW fits into their overall HIV prevention landscape and how to address challenges associated with its introduction.

ered AIDS-free, Mentored, and Safe) initiative-a partnership between the U.S. President's Emergency Plan for AIDS Relief (PEPFAR), the Bill \& Melinda Gates Foundation, and Girl Effect, plus Johnson \& Johnson, Gilead Sciences, and ViiV Healthcare ${ }^{9}$-aims to significantly reduce new HIV infections among AGYW in 10 countries in sub-Saharan Africa by providing a core package of interventions to AGYW residing in areas with high HIV prevalence. The provision of PrEP is currently part of the prevention package for some of the DREAMS countries, and is being considered by several others. Under the DREAMS mandate and other global efforts, new programmatic strategies for introducing this innovative product in real-world settings are being developed and implemented. There is an urgent need for implementation science to complement biomedical, clinical, and product marketing research in order to appropriately introduce PrEP to AGYW.

The equitable and ethical introduction of this new biomedical intervention has to take into account the particular vulnerabilities that AGYW may face; these include, but are not limited to, legal and policy constraints affecting access and ability to consent to sexual and reproductive health services, stigma around AGYW sexuality, power dynamics between AGYW and parents and partners, experiences of violence and coercion, and individual capacity to assess HIV risk. ${ }^{10}$ Further, the past introduction of a number of health technologies for women (e.g., human papillomavirus vaccine, emergency contraception) have highlighted women's concerns around how a product works, whether it has the potential to cause harm, whether it will jeopardize fertility, and whether it will influence relationships. ${ }^{11} \mathrm{As}$ countries consider and plan to introduce and expand PrEP implementation under DREAMS and other initiatives, there is a need to carefully examine the individual behavioral factors as well as the structural and sociocultural factors that may influence PrEP acceptability, uptake, and adherence among AGYW. Countries also will need to develop a vision for how PrEP for AGYW fits into the overall HIV prevention landscape and how to address challenges associated with its introduction, ${ }^{11}$ such as determining the:

- Most appropriate sub-populations of AGYW for using PrEP, given the nature of the HIV epidemic within the country. ${ }^{12}$

- Feasibility of introducing PrEP to AGYW in a safe and ethical way that supports their human rights.

- Ability of health services to reach the most vulnerable AGYW.

- Influence of service providers, partners, families, and communities on AGYW's acceptance of PREP.

- Willingness of AGYW to utilize and adhere to PrEP.

- Policy and service delivery issues that arise in the early stages of new product introduction.

- Best ways to introduce PrEP to AGYW into existing health care systems.

\section{PURPOSE OF THE DOCUMENT}

This document was developed by the Population Council, with support from the Bill \& Melinda Gates Foundation, to provide DREAMS country teams with practical guidance on building evidence to guide PrEP introduction for AGYW. 
The primary audiences for this document are health policy makers and program planners who will be making decisions about how PrEP is introduced to AGYW and the researchers who will assess AGYW's specific needs and experiences.

Our aim is to complement emerging global guidance on $\operatorname{PrEP}^{1}$ and on-going work regarding delivery platforms, marketing, and policy and regulatory frameworks for PrEP introduction more generally. We focus on examining the factors that influence informed choice, demand, and use of PREP by young women and that influence client-provider interactions. Further, we hope this document can serve as a useful guide to gather data on user, community, and provider perspectives as countries move from introduction to broad-based implementation of PrEP.

This practical guidance document is informed by the Population Council's work on HIV prevention product introduction for women ${ }^{11,12}$ and builds on our commitment to understanding whether, when, how, and under what circumstances ARVbased prevention would be beneficial for AGYW.

\section{ETHICAL CONSIDERATIONS}

Depending upon the types of data to be collected, and for what purpose (for program development versus research publication), the data collection proposed in this document may need to undergo an ethical review, may be exempt from an ethical review, or may be considered to not be research (and therefore not require an ethical review). As guidelines for ethical reviews vary by setting and organization, it is recommened that you check with your local ethical/institutional review board to determine the best way forward.
Regardless of whether the data collection proposed in this document must undergo an ethical review, it is the responsibility of the research team to fulfill several obligations and ensure safeguards in planning and conducting the research, as well as reporting research findings. Specifically, ethical standards need to be met for maintaining the dignity and welfare of all participants; protecting them from harm, unnecessary risk, or mental and physical discomfort; and ensuring that respondents are not being unduly influenced or induced into participation in the research. Further, ethical guidelines pertaining to the accurate and appropriate use of data should be maintained by researchers and others using the data. These issues are particularly important to consider when conducting research with minors (those below the age of legal consent) and marginalized populations.

Several available resources provide guidance on research with minors and should be consulted during the design and development of this research. Some examples include http:// childethics.com and http://www.popcouncil.org/ uploads/pdfs/horizons/childrenethics.pdf.

Before data collection, all participants should be given information on PrEP attributes. UNAIDS' "Oral pre-exposure prophylaxis-Questions and answers" is a brief resource guide (published in 2015) that contains answers to pertinent questions about PrEP, its use, and implementation. The guide can be downloaded at http://www. unaids.org/en/resources/documents/2015/ Oral_pre-exposure_prophylaxis_questions_and_ answers. The information given should cover who is eligible to take PrEP, route of administration, frequency of use, side effects, and the need for HIV testing.

${ }^{1}$ The WHO is currently developing comprehensive global implementation guidelines for PrEP. 


\section{STRUCTURE OF THE DOCUMENT}

This guidance document is divided into five sections, as follows:

Section 1: Provides a framework for PrEP introduction to AGYW.

Section 2: Focuses on the key actors that influence AGYW's choices-AGYW themselves, service providers, male partners, parents and guardians, and policy makers, respectively. For each type of actor, the section provides a brief rationale to reaching this key actor, important domains to investigate for guiding PrEP introduction, recommended data collection methods, and illustrative questions per domain.

Section 3: Describes additional data collection techniques and methods to gain a deeper perspective of participants' views.

Section 4: Provides practical advice for conducting analysis.

Section 5: Provides an overview of practices to foster research utilization.

4 - Building evidence to guide PrEP introduction for adolescent girls and young women 


\section{SECTION 1}

\section{DEVELOPING A PREP INTRODUCTION STRATEGY FOR AGYW: A CONCEPTUAL FRAMEWORK}

In this section we present a framework (Figure 1, page 7) that highlights the range of players, relationships, and contextual factors that can affect AGYW's health-seeking behaviors and their PrEP uptake and use. This framework is useful to consider when designing PrEP introduction strategies. It positions young women in the context of their partner, peers, family, health providers and services, social values/community context, and broader scientific knowledge about PrEP-all of which can interact and influence, sometimes positively and sometimes negatively, the choices that AGYW make or are able to make. ${ }^{2,10,13-17}$

The framework consists of two sides that interact, demonstrating the intricacies and dynamic aspects of PrEP introduction among AGYW. The right side of the framework focuses on the main factors that determine the feasibility of effective introduction of quality PrEP services for AGYW.

These include the scientific context, political landscape, health system infrastructure, and provider dynamics. For instance, regulatory guidelines that identify target populations for PrEP can have major implications for who can access PrEP. Similarly, decisions around consumer drug costs and service delivery platforms can have important implications for AGYW. Service providers are gatekeepers of new medical products and their knowledge, attitudes, and perceptions will determine whether and how they offer PrEP to young women as an HIV prevention method. An as- sessment of these factors will determine whether health systems have the capacity to deliver PrEP safely and effectively to AGYW.

The left side of the framework focuses on the main factors that determine whether young women are able to make informed decisions around PrEP uptake and its effective use. Key factors that influence informed choice, uptake, and use of PrEP include the community context, family dynamics, partner dynamics, and AGYW characteristics. For instance, broader community-level knowledge about PrEP, parental consent for PrEP use, and experiencing violence within sexual relationships are likely to influence AGYW's decisions about PrEP. Further, young women's access to education, information, and social support, and their own HIV risk perceptions are likely to significantly influence PrEP uptake and use. Examining these factors will highlight key barriers and facilitators to PrEP uptake, use, and adherence among AGYW.

The two sides of the framework are connected to illustrate the interplay between them, which we need to fully understand if PrEP for AGYW is to be realized. AGYW and service providers are depicted at the center of the framework to demonstrate the relative importance of provider-AGYW interactions. Consequently, it will be important to engage both service providers and AGYW in developing programs for PrEP introduction. 
The factors and relationships depicted in the framework extend beyond the initial phases of PrEP introduction and should be examined as part of ongoing efforts to monitor safety, adherence, and service quality.

6 - Building evidence to guide PrEP introduction for adolescent girls and young women 


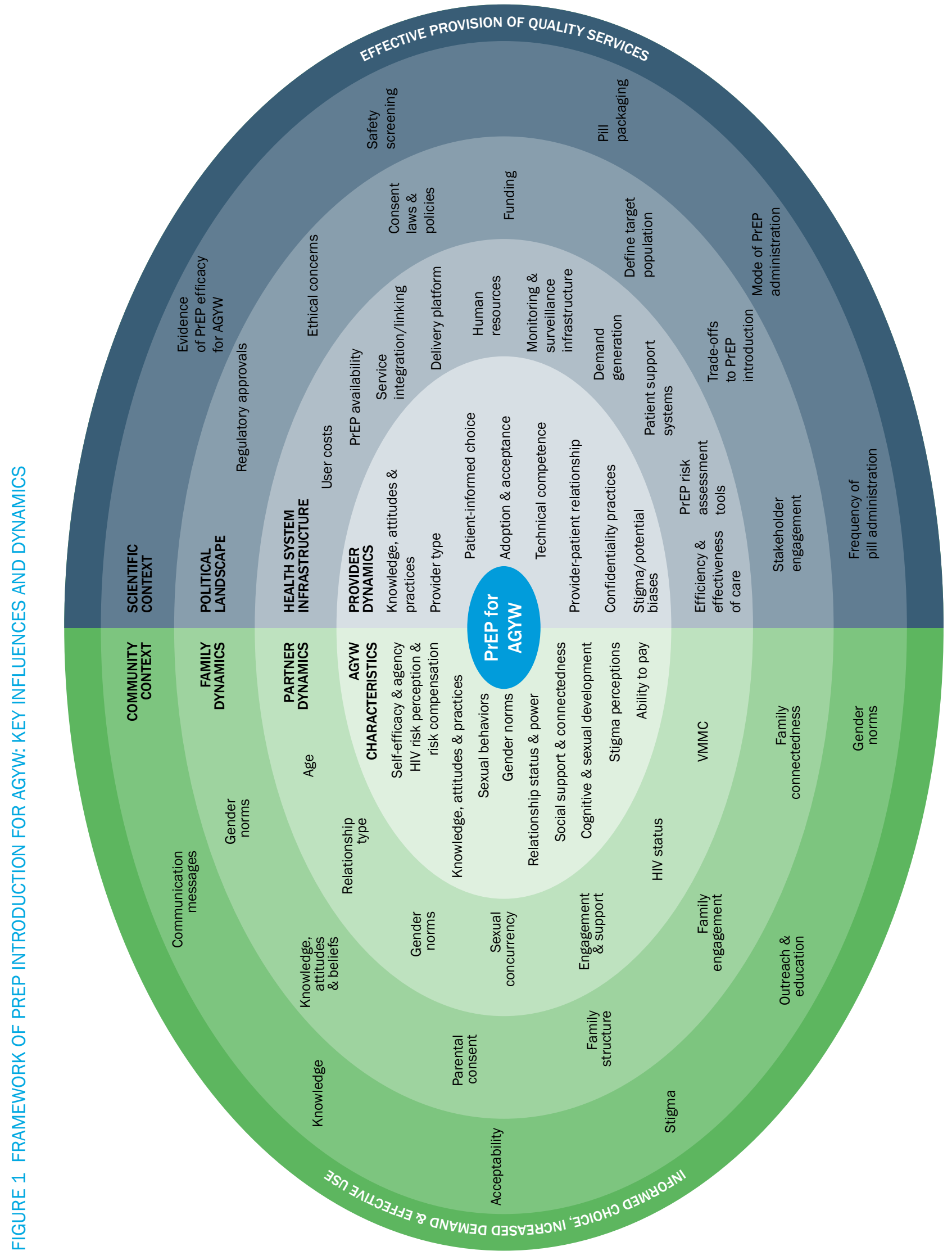




\section{SECTION 2 ENGAGING KEY ACTORS: KEY DOMAINS, METHODS, AND QUESTIONS}

\section{ADOLESCENT GIRLS AND YOUNG WOMEN}

The success of PrEP as an HIV prevention intervention among AGYW is largely dependent on its successful uptake and adherence by AGYW, the end-user of the drug. A critical assessment of AGYW's knowledge, attitudes, perceptions, current sexual and reproductive health behaviors, social context, and engagement with the health care system will aid in identifying and reaching AGYW who are most at risk of acquiring HIV, determing challenges associated with acceptance and uptake, and identifying opportunities to support AGYW in protecting themselves against HIV.

The assessment needs to start with clarity around the AGYW sub-population to be reached. Once the focus AGYW group is decided, then the specific actors who might play a role in influencing those AGYW's decisions about PrEP can be effecively identified.

This section provides guidance on how to undertake such an assessment to inform a PrEP introduction strategy for AGYW that reduces barriers while enhancing facilitators to uptake and adherence.

\section{Population}

HIV vulnerability varies within the population of AGYW. For example, studies have shown that female sex workers (FSWs) are at a considerably higher risk of acquiring HIV when compared to the general AGYW population. ${ }^{18}$ The nature of the epidemic will determine relevant sub-populations of AGYW in a given setting. Program planners are encouraged to utilize existing epidemiological evidence and literature to identify the subpopulations who are the most vulnerable to HIV within their setting and who should be included in an assessment to understand the barriers and facilitators to uptake and adherence.

An understanding of these sub-populations' barriers and facilitators aids in tailoring a PrEP introduction strategy. See Box 1 for potential sub-populations to include in an assessment.

\section{Key Domains}

PrEP Attributes, Acceptability and Potential Use: There are several attributes of PrEP, such as frequency and route of administration, side effects, and the need for frequent HIV testing, that may influence AGYW's likelihood to use and adhere to it. Additionally, there is a need to assess AGYW's attitudes and perceptions of PrEP after learning about how it works. The evidence shows that 


\section{BOX 1 \\ Potential sub-populations of AGYW}

Female sex workers

AGYW who engage in transactional sex

AGYW who engage in cross-generational sex

AGYW with multiple concurrent partners

Married AGYW

Orphaned AGYW

Out-of-school AGYW

AGYW living in hotspots

AGYW with HIV-positive sexual partners

AGYW who perceive themselves to be at high risk of HIV acquisition

users gain the greatest benefit of PrEP with very high adherence. ${ }^{4}$ Thus, understanding the factors, such as side effects, testing requirements, and drug supply, that are likely to influence adherence are also important to assess.

Risk Perception and Compensation: Using and adhering to PrEP is influenced by AGYW's perceptions of their risk for acquiring HIV; if an AGYW believes she is at low risk of acquiring HIV, it is unlikely that she will access, use, and adhere to PrEP. On the other hand, there is concern that AGYW who perceive themselves at risk of acquiring HIV and do use PrEP, will increase risky sexual behaviors and reduce existing risk-reduction behaviors, which is known as "risk compensation." ${ }^{19}$ There is little evidence of risk compensation, but understanding AGYW's risk perceptions and potential for risk compensation prior to PrEP introduction can aid in developing strategies to make risk salient to AGYW and maximize motivations for prevention. ${ }^{2}$

Gendered Power Dynamics: Inequitable gender norms and gendered division of power within sexual relationships have been shown to play an important role in AGYW's ability to accept and adopt sexual and reproductive health interventions, including contraceptive use and condom use. ${ }^{2,20}$ Thus, PrEP introduction strategies should take into account how gendered power dynamics and control over decision-making may influence perceived risks and benefits of PrEP, which impacts uptake and adherence.

Stigma and Social Acceptability: The willingness of AGYW to use and adhere to PrEP will greatly depend on social understandings, including whether it is seen as an effective, healthy option and a socially acceptable strategy for HIV prevention. ${ }^{21}$ Additionally, stigma has been shown to influence PrEP uptake and adherence in a few studies because PrEP use might lead others to believe users are engaged in high risk sex or are HIV positive since PrEP is an antiretroviral. ${ }^{21}$ Understanding PrEP-related stigma and social acceptability in a given setting is important for designing introduction strategies for AGYW.

Service Delivery Models and Strategies: The successful uptake of PrEP by AGYW will be dependent on the service environment where it is offered and whether AGYW feel comfortable accessing services from these locations. PrEP introduction strategies should consider the feasibility of offering PrEP in diverse settings based on AGYW's preferences.

\section{Methods}

We recommend two methods to assess the challenges and opportunities associated with PrEP acceptance, uptake, and adherence among HIV-negative, sexually active AGYW: focus group discussions (FGDs) and in-depth interviews (IDIs). 
Use IDIs to gain information on topics that are sensitive in nature, such as power dynamics within sexual relationships, and when seeking information about personal perceptions, attitudes, and behaviors. For each sub-population, a minimum number of IDls is four to six. Program planners might also consider if groups should be conducted in various geographical regions in order to gain a broader overview of barriers and facilitators.

Use FGDs to generate broad overviews of PrEP acceptance, uptake, and adherence to gain information about social factors that would influence PrEP acceptance, and to brainstorm and generate ideas on how to increase acceptability and uptake. Consider conducting FGDs after a few IDIs have been completed to determine whether some themes from the IDIs are present within a larger group of AGYW. For each sub-population, an ideal focus group size is between 6 and 10 participants and the assessment should include at least two to three groups of participants. Again, program planners might also consider if groups should be conducted in various regions in order to gain a broader overview of barriers and facilitators.

Eligible AGYW are those who are HIV-negative. To draw the sample, purposefully select AGYW from a variety of settings where specific sub-populations are found in order to gain comprehensive information. Partnering with community-based organizations (CBOs)/nongovernmental organizatons (NGOs) who work with and are trusted by the different sub-populations of AGYW can provide detailed insights into where and how to recruit respondents. Healthcare settings, such as antenatal and family planning clinics, are one easily accessible location to recruit AGYW. AGYW recruited from these sites can also assist with the recruitment of their peers who might not access these services. Venues where AGYW are known to frequent can also serve as good recruitment sites, such as CBOs, schools, youth clubs, markets, shopping centers, bars, clubs, and large community events. The staff of NGOs can provide insights into where sub-group populations, especially marginalized populations, such as young FSWs, could be reached. Another approach that works well with marginalized populations is snowball recruitment or sampling. This entails the interviewers asking the participants of initial IDIs and FGDs to help connect them to their peers.

Ideally, FGDs and IDIs should be conducted by trained facilitators who are drawn from the participating sub-population or by individuals who can develop good rapport with participants, such as staff of CBOs. For breadth and depth, AGYW participating in IDIs should not participate in FGDs. Rather, the research team should assess the comfort level of each potential participant to determine whether to assign her to an FGD or an IDI. FGDs should be homogenous within sub-populations of AGYW; that is, all participants for each FGD should be a member of the same sub-group (e.g., only FSWs should be in a FSW FGD). It is also important that FGDs and IDIs are conducted in safe spaces to ensure safety and confidentiality.

While these data collection strategies will inform how to effectively introduce PrEP to AGYW, continued monitoring of the domains after PrEP introduction will be needed to determine uptake and adherence. As such, a monitoring plan should be developed.

\section{Questions}

Note: Some of the questions noted below will not be appropriate for group discussions due to their sensitive nature.

\section{PrEP attributes, acceptability, and potential use}

- Had you heard about PrEP before today? (If a FGD, ask for a show of hands and record number) If so, what had you heard, and who 
or where did you hear it from? (Probe for health care provider, school, community-based educator, family/friends, PrEP user)

- What do you see as the benefits of PrEP for you personally?

- What about for other young women like you?

- In order for PrEP to work, people have to take a pill every day. What do you think about having to take a pill every day?

- What do you think other young women like you would think about taking a pill every day?

- Another way that is currently being explored for people who want to take PrEp involves taking a series of pills right before having sex and then continuing to take pills shortly after having sex. What do you think about this method of taking PrEP, which provides protection just for that/those sexual encounter(s). Which of these ways are you more interested in, and why?

- Of the two methods-taking a pill every day or taking it before/after sex-which method would you prefer, and why?

- Which method do you think young women like you would prefer and why?

- Based on what you learned today/have heard before, how would the following things about PrEP influence your decision and that of other young women like you about taking PrEP? (Facilitators can also lead the respondents through a listing and ranking exercise to identify major and minor concerns.)

- The level of effectiveness in preventing HIV transmission

- Having to take a pill every day

- Possible side effects of PrEP (which ones?)

- Having to pick up a supply on a regular basis

- The requirement to be tested for HIV every two to three months
- The requirement to receive counseling about adherence on a regular basis (adherence means taking medications in the correct amount, at the correct time, and in the way they are prescribed)

- The color of the pill

- Having to store or keep the pills

- The cost of the pill

- Any other concerns

- How would it make you feel to start taking PrEP? (Please describe)

- Are there any cultural traditions in your community(ies) about taking medications that would make young women more or less likely to take PrEP? (Describe)

- What if you were given something to motivate you to take PrEP?

- What should the incentive be? (Probe for money, airtime)

- If PrEP became available in your community in the next week, how willing would you be to start taking it immediately?

- What if PrEP became available within the next six months, how willing would you be to start taking it immediately?

- Earlier we talked about certain aspects of PrEP, including possible side effects and requirements to be tested, receiving adherence counseling, and picking up a pill supply on a regular basis. Do you think any of these things would make it more difficult to continue to take PrEP every day, over months or years? Which? (please describe)

- If you started to take PrEP, what would help you continue to take it every day, over months or years? (Probe and ask participants to rank from most to least helpful for: close location of PrEP services, support from partner, support from friends and family, other people you know taking PrEP, mobile phone text or call reminders, attitude of health facility staff, lower cost.) 
- How easy or difficult do you think it would be to incorporate taking a daily pill into your current routine? Why?

- What factors would affect your ability to take PrEP on a daily basis? What factors would enhance your ability to adhere to a daily PrEP regimen? (Probe: product storage, dosing when away from home, peer support, other support measures like SMS reminders or adherence counseling, fear of others finding out or of GBV.)

\section{Gendered power dynamics}

- Please tell me about instances where you have discussed avoiding/preventing HIV with your primary sexual partner? (Probe on condom use)

- Do you have additional sexual partners, and if so how many?

- Does your primary sexual partner have other sexual partners (that you are aware of)?

- How do your sexual partner's opinions about you using products (e.g., condoms, contraceptives) to prevent HIV and pregnancy affect your decisions to use them?

- How might you discuss your desire to start taking PrEP with your sexual partner?

- How would your primary sexual partner's opinion about PrEP affect your decision to take it?

- What if he agrees? What if he disagrees?

- Is your sexual partner currently taking PrEP?

- If you considered taking PrEP without telling your primary sexual partner, what impact might this have on your relationship?

- How might he react if he found out?

- What would be some reasons why you would not tell your partner that you are interested in taking PrEP or you were taking PrEP?

\section{Risk perception and risk compensation}

- How likely do you think it is that you will get HIV? Why? (Probe for number and types of sexual partners, frequency of sex, condom use, knowledge of HIV status of partner)

- How might your concerns about getting HIV, influence your interest in starting to take PrEP?

- How might using PrEP change the type and number of sexual relationships you have?

- How might the use of PrEP influence condom use with your sexual partners?

\section{Stigma and social acceptability}

- What do you think your friends know about PrEP? How might their opinion about PrEP influence your decision to use PrEP?

- What do you think your parents/guardians know about PrEP? How might their opinions influence you?

- What about other leaders in your community (e.g., teachers or religious leaders)? What kind of influence do they have on your opinions and decisions about PrEP?

- How do other people in your community think about ARVs and people who take ARVs? How do they treat people who take ARVs?

- In what way would these views influence your decision about whether to use PrEP (If so, how)?

- If you knew a friend was taking PrEP, what would you think of her?

- If you started to take PrEP, how likely would you be to share this information with your peers (or family members)? What factors would encourage/discourage you to do so?

\section{Service delivery strategies and models}

- Where would you prefer to go for PrEP services and to collect PrEP pills? (Probe for health clinic, ARV clinic, youth-friendly clinic/service, 
pharmacy, private provider, family planning clinic, NGO) What are the pros and cons of each place?

- What do you think about the idea of PrEP being delivered outside of a health facility (such as at school or at your home)? What might be the barriers and benefits to this different approach?

- What is the best way to reach adolescent girls and young women with messages about their sexual health (Probe for radio, television, peer educators, health care providers, posters, flyers, social media, cell phone texts, etc.)?

- If you were going to create a campaign to encourage young women like yourself to consider using PrEP to reduce their risk of getting HIV, how would you describe what PrEP is and how it works (in your own words)?

- What information about PrEP should be emphasized in communication campaigns? What should not be emphasized?

- For young women who start on PrEP, what is the best way to encourage them and remind them that they need to take the medication and to get tested for HIV every few months? (Probe for phone call reminder, text message reminder, letter) 


\section{SERVICE PROVIDERS}

Service providers of varying skill levels and service settings serve as the gatekeepers of new technologies requiring a prescription. PrEP introduction efforts depend on service providers and the health systems within which they work having the capacity to deliver the product safely and effectively to AGYW. A critical assessment of service providers' knowledge, attitudes, beliefs, concerns, competencies, and behaviors is needed in the following areas: 1) the introduction of a new technology into their service system and ability to provide quality care; 2 ) the introduction of an ARV-based product to AGYW; and 3) the provision of sexual and reproductive health services to AGYW in general.

This section provides guidance on how to undertake such an assessment to determine effective provision of quality PrEP services for AGYW. This assessment should provide insight into what strategies are needed for successful introduction and ultimately, uptake and adherence by AGYW.

\section{Population}

The assessment should be conducted with appropriate cadres of service providers in a variety of health service settings to ensure appropriate counseling, provision, testing, resupply, and follow-up of AGYW. The assessment should generate insights from a variety of service providers who may be eligible to provide PrEP to AGYW. The selected providers will vary in a given setting. A potential list of service providers to include in the assessment is provided in Box 2.

It also is important to determine which service delivery settings would be most appropriate and feasible to reach different sub-populations of AGYW with PrEP. Therefore, service providers included in the assessment should be drawn from the diverse service delivery settings that exist
BOX 2

Potential health service

providers

Clinic administrators

Doctors and medical officers

Private medical practitioners

Nurses

Counselors

Lab technicians

Outreach workers

Pharmacists

Peer educators

within a given context. A potential list of service settings is provided in Box 3.

The information gathered from service providers and AGYW can help determine the feasibility of introducing PrEP, and if feasible, the best service delivery settings and service providers to provide PrEP.

\section{Key Domains}

PrEP Knowledge, Attitudes and Perceptions: Recognizing the gatekeeper role that service providers play in influencing service delivery and uptake, it is important to assess their knowledge, attitudes, and perceptions. Training on the technical aspects of PrEP introduction and use can be undermined by providers who hold negative attitudes and viewpoints toward PrEP. Existing research, mainly from developed country settings, shows that providers have a number of concerns that would influence their prescribing 


\section{BOX 3}

Potential service settings for

\section{delivering PrEP}

Public-sector health centers and hospitals

Private medical practice health centers and hospitals

Non-governmental organizations

Faith-based organizations

University-based health clinics

Youth-friendly health service locations

HIV counseling and testing sites

STI prevention and treatment sites

Family planning \& reproductive health sites

Maternal health settings

Pharmacies

Gender-based violence counseling sites

Mobile health services

of PrEP, including drug resistance, drug-related toxicities, the potential for funds to be diverted from HIV-related behavior change programs, and risk compensation. ${ }^{11,21}$ Thus, the findings from an assessment will help program planners determine the providers' training needs to make PrEP introduction effective.

Stigma: Stigma from service providers within service delivery settings is an important component to assess and understand. ${ }^{21,22}$ The contraception literature shows that service providers serve as barriers to uptake and use because many refuse to counsel about and provide contraception to adolescents due to their attitudes and beliefs about adolescent sexual activity. ${ }^{22}$ Therefore, it will be necessary to address stigma within service delivery settings to reach and protect AGYW if PrEP introduction is to be feasible and successful. A failure to assess and subsequently address service providers' biases can significantly impede AGYW access and uptake of PrEP.

\section{Legal and Ethical Considerations: There are} many ethical and legal considerations that must be assessed so that those at high risk of acquiring HIV and most likely to benefit from PrEP can access this promising intervention. For example, in some settings adult consent is required in order for adolescents under the legal age of consent to receive certain HIV services and treatment; PrEP is likely to be included in the list. ${ }^{10}$ The breach of the child's right to confidentiality and access to care (as many vulnerable youth do not feel comfortable disclosing their behaviors to adults) are among the ethical concerns surrounding the need for adult consent. Safety is both a legal and ethical concern as some behaviors are illegal (e.g., selling sex) and AGYW may avoid accessing services. Another example is the obligation of service providers to report adolescents who are below the legal age of consent requesting PrEP or to report behaviors of adolescent key populations in settings where certain behaviors are illegal. ${ }^{10}$ Therefore, service providers' knowledge of existing consent laws and other legal considerations, how they interpret and implement laws, how existing laws affect the provision of services to AGYW.

Service Delivery Considerations: PrEP introduction and ultimately the potential benefits it may offer to AGYW may be severely undermined if health services lack the capacity and the ability to meet the technical requirements of PrEP. ${ }^{11,22}$ These include the existence of written medical guidelines, protocols, and standards at the service delivery sites; the possession of necessary equipment and supplies (e.g., HIV test kits); existence of adequate space to store products; and having the human resources to provide PrEP. It is also important to gain service providers' insights 
into which service delivery settings are most appropriate and prepared to provide PrEP to AGYW to increase likelihood of acceptance, uptake, and adherence.

Service Access and Quality of Care: Provider-patient interactions influence service access and quality of care provided. Adherence is a particularly challenging aspect of PrEP and there is need to develop strategies to effectively support and measure adherence. ${ }^{11}$ Provider counseling of AGYW on correct and consistent use of PREP is a key strategy for fostering adherence. ${ }^{23,24}$ Therefore, the existing capacity of service providers and service delivery systems to provide high quality counseling needs to be assessed. These results will inform the training of providers, and can be particularly helpful in dissipating providers' reluctance to adopting new interventions that require special counseling and educational skills they do/may not possess. ${ }^{23,24}$ Outside of counseling, a general assessment of how providers interact with AGYW is important to understanding how to improve the quality of care provided. To create the most effective training, the assessment should solicit providers' insights into the best counseling messages for AGYW and their own training needs. ${ }^{23}$

\section{Methods}

We recommend two strategies to assess the capacity of health care providers within different service delivery systems for PrEP introduction and roll-out: IDIs and surveys. Use IDIs to gain information on individual service provider's knowledge, attitudes, beliefs, concerns, perceived competencies, and experiences providing care to AGYW in a variety of health service settings. IDIs are preferred to FGDs because they allow for the exploration of sensitive (e.g., personal beliefs about adolescent sexuality, experiences with youth clients) and non-sensitive (e.g., service delivery staff requirements for PrEP) topics.
Furthermore, the interviews can provide in-depth information on factors relevant to distinct service delivery settings, such as client flow, patient intake systems, and referral mechanisms. A minimum of two to three IDIs should be conducted per service provider type per service delivery setting type. Program planners must also consider regional variations.

One good option is to recruit and train service providers currently offering HIV services to facilitate the IDIs because they will be knowledgeable and aware of the critical issues in HIV prevention in their country. Being interviewed by peers who understand their daily experiences will also encourage service providers to be honest about their perspectives of PrEP. To encourage service providers to participate in the interviews, ensure confidentiality of their responses by anonymizing their transcripts and assure them that responses provided will not jeopardize their employment.

To complement the richness of the IDIs, the assessment should also include a survey administered to a large cross-section of service providers in a variety of service delivery settings. The survey should collect information on many of the same topics covered in the IDIs. Consider conducting the survey after the IDIs are completed so that the survey design is informed by the results from the IDIs. It will also allow program providers to determine whether the findings from the IDIs are present in a larger sample of providers. Given that this population is likely to have high literacy levels, surveys can be self-administered. The sample size for the survey will depend on the number of facilities capable of offering PrEP and the number of providers at these sites in each region of interest to program planners. One option is to administer the survey to all providers within a selected number of facilities. However, consult with a statistician to determine the sample size needed in order for findings to be generalizable to a type of service provider and service deliver setting. 
After the assessment, program planners should consider conducting a one to two day workshop with service providers from a variety of service delivery settings to discuss the findings from the IDIs and survey. This format is conducive to building consensus among service providers on the feasibility of PrEP introduction and allows participants to work together to develop locally grounded strategies to build capacity for offering PrEP. Participants can be divided into breakout groups to discuss questions about PrEP that arise. A note taker for each group is important to record all main points. Following the breakout sessions, groups should be brought back together to report on their discussion and start the consensus building process.

\section{Questions}

A 2012 consultation led by the Population Council on "Shaping the operations research agenda for antiretroviral-based prevention products for women" identified several important domains for operations research relevant to PrEP and service providers, including service delivery models, programs and services; providers; type and training; HIV testing and retesting; and potential resistance. Several questions presented below are drawn from this document. We encourage program planners to access the document for more detailed information at http://www. popcouncil.org/uploads/pdfs/2012HIV_ShapingORAgendaARV.pdf.

We also encourage program planners to access another Population Council resource, "ARVbased HIV prevention for women: State of the science and considerations for implementation in Zimbabwe. Report from a provider workshop," to aid in designing a workshop with providers focused on PrEP and applicable questions that can be included. This resource can be downloaded at: http://www.popcouncil.org/uploads/ pdfs/2015HIV_ZimbabweProviderWorkshop.pdf.

\section{Qualitative questions}

PrEP knowledge, attitudes and perceptions

- What have you heard about PrEP?

- Have you received any professional or technical guidance/training regarding PrEP, from the government or international organizations? (If yes, please describe)

-What do you see as the potential benefits of introducing PrEP?

- What concerns, if any, do you have about introducing PrEP? (Probe on safety, efficacy, drug resistance, risk compensation)

- Who should have access to this new intervention?

- Which groups would most benefit from PrEP?

- For each group that would benefit, where and how would they best be reached?

- Would you feel comfortable prescribing PrEP?

- How might you assess if a patient/client needs might be a good candidate for PrEP?

- What additional information would you need in order to feel comfortable prescribing PrEP?

- Based on your experiences, what are your opinions about making HIV PrEP available for AGYW?

- How would you feel about prescribing PrEP to AGYW?

- What do you see as the potential benefits of introducing PrEP for AGYW?

- What are the drawbacks?

- What additional information would you need in order to feel comfortable providing PrEP to AGYW or certain subpopulations of AGYW?

- Under what circumstances would you prescribe PrEP to AGYW? 


\section{Legal and ethical considerations}

- What type of legal/ethical issues do you currently encounter when providing HIV or other types of sexual and reproductive services to adolescents and young adults? (Probe on sub-groups of AGYW such as FSWs, orphans, married AGYW)

- How do you address these issues?

- What actions should policy makers take to address these issues?

- If in the next year, the government endorsed/ provide PrEP, how would that influence your opinion of PrEP?

- What legal or ethical issues would consider in providing PrEP to young women?

- How do national regulations regarding the provision of ARV-based medications to AGYW influence your work?

- What are current parental consent laws around HIV services for young people?

- How would this affect protocols for providing PrEP to AGYW at your clinic/ health facility?

- Under what circumstances should AGYW be able to consent for themselves? Why these circumstances?

- What kind of information do you think should be provided to partner(s) or parent(s)/guardian(s) of AGYW who are on PrEP or seeking PrEP?

- What is the obligation of the health care provider in reaching the AGYW's sexual partner(s) or parent(s)/guardian(s)?

- How do you think community leaders would respond to the introduction of PrEP for AGYW? What about religious leaders or institutions? Schools? Other groups?

\section{Service delivery considerations}

- What do you consider appropriate service delivery settings to offer PrEP to AGYW?
- What cadre of staff (i.e., nurse, clinical officer, health worker) would best be able to screen, counsel, and prescribe PrEP to AGYW?

- At your clinic/health facility, what kinds of trainings might be needed on the provision of PrEP? And on being able to provide PrEP to AGYW?

- At your clinic/health facility, how would providing PrEP affect existing services in terms of client flow, operations, staffing, storage, etc.?

- What do you foresee as the key challenges providers may face in offering PrEP? And in offering PrEP for AGYW?

- How can you support women's use of the PrEP or other daily ARV-based medication? In what ways do you help women understand and manage potential side effects?

- How do you/your clinic, currently measure/ assess if clients are adhering to their ARV medications?

- What challenges would AGYW face in adhering to PrEP?

- In your experience, what strategies have been effective for supporting adherence among PrEP users?

- What are the storage implications for PrEP at the service delivery site?

- What service model package is most effective for reaching various sub-populations of AGYW?

- Which program entry points would be most effective to deliver PrEP?

- ARV-based prevention products will require counseling, HIV testing, and monitoring. Which service settings might afford good opportunities for delivering PrEP? (Probe on existing settings in the country)

\section{Service access and quality}

- What are the main challenges that AGYW might face in getting access to PrEP? 
- How well do you feel your clinic/health facility is currently doing in providing the following services, and for each, how can service delivery be improved?

- Quality of care overall

- Quality of care for HIV-positive patients on ARVs

- HIV testing and counseling

- Adherence counseling, for ARVs and other regimes requiring adherence

- SRH services for adolescents

- Welcoming and comfortable environment for AGYW

- If PrEP was introduced in the country, how do you think this would affect existing HIV prevention and treatment programs and the health system more broadly? (Probe on HIV testing programs, HIV treatment programs, sexual and reproductive health programs/ services, staff deployment, task-shifting)

- Are there lessons (positive and negative) from the introduction of other technologies or HIV prevention strategies that could inform plans for introducing PrEP?

- Are there lessons (positive and negative) from other HIV prevention programs with AGYW that could inform plans for introducing PrEP for AGYW?

- In your opinion what might be the best way to create demand among AGYW for this new product?

\section{Stigma}

- Who do you consider to be the most at risk for HIV? Why? (Probe on AGYW and sub-groups)

- How well do you think AGYW are able to assess their level of HIV risk? How do you counsel youth clients who think they are at high risk of HIV?

- How do providers currently view AGYW who take ARVs? Does this differ by the client's age? How they acquired HIV? Reported sexual activity?

- Why might community groups have negative reactions to providing PrEP to AGYW?

- Why might health care providers have negative attitudes about providing PrEP to AGYW?

- How would you describe your feelings about prescribing PrEP to AGYW? Would this differ by the client's age (girls age $10-14$ vs. $15-19$ vs. 20-24), and if so, how?

- How do you think you would react if an AGYW client requested PrEP?

\section{Quantitative questions}

The quantitative survey should collect provider demographic information to assess whether PrEP knowledge, attitudes, and experiences differ by provider demographics, such as age, gender, medical training, facility type, experience caring for HIV-positive patients, or perceived HIV risk.

\section{PrEP knowledge, attitudes and perceptions/ stigma}

Response categories for the following questions are: (a) strongly agree; (b) agree; (c) neutral; (d) disagree; and (e) strongly disagree.

Provider attitudes and perceptions about PrEP in general

Note: The questions in this section were adapted from Mimiaga MJ, et al. 2014. "Suboptimal awareness and comprehension of published preexposure prophylaxis efficacy results among physicians in Massachusetts," AIDS Care 26(6): 684-693. ${ }^{30}$

1. There is not enough data on PrEP's clinical efficacy

2. I am concerned about the potential side effects of PrEP

3. I do not have time to provide clinical monitoring 
4. I do not have time to engage in prevention counseling

5. The use of PrEP will cause patients to engage in riskier behaviors

6. The provision of PrEP will result in an increase in sexually transmitted disease incidence among patients

7. The use of PrEP will result in less frequent HIV testing among patients

8. Non-biomedical HIV prevention interventions (behavioral) are more effective than PrEP

9. Non-biomedical HIV prevention interventions (behavioral) are safer than PrEP

10. The use of PrEP for prevention will result in less funding for HIV treatment

11. Antiretrovirals for prevention will select for, and disseminate, drug resistance

12. Non-biomedical HIV prevention interventions (behavioral) should be attempted before prescribing PrEP

13. I am uncomfortable prescribing drugs for new indications unless there are many years of follow up data

14. I believe PrEP should be readily available to all persons at risk for HIV infection

15. I would provide PrEP to the following subpopulations [record response for each]

a. High-risk men who have sex with men

b. Injection drug users

c. People with a sexually transmitted disease

d. People who change sex partners frequently

e. People who exchange sex for money, drugs or other goods

f. AGYW

g. Discordant couples

h. Any person who believes he/she is at high risk for HIV infection i. Any person regardless of risk

Attitudes and perceptions about PrEP for AGYW

1. AGYW are at high risk for acquiring HIV

2. PrEP should be readily available to AGYW

3. There are certain sub-populations of AGYW who are at high risk for acquiring HIV

4. PrEP should be readily available to certain sub-populations of AGYW

5. The use of PrEP will cause AGYW to engage in riskier behaviors

6. Administering PrEP to AGYW would result in backlash from the community

7. Administering PrEP to AGYW would result in backlash from religious leaders

8. I would provide PrEP to the following subpopulations of AGYW [record response for each]

a. AGYW with a sexually transmitted disease

b. AGYW who change sex partners frequently

c. AGYW who exchange sex for money, drugs or other goods

d. AGYW with an HIV-positive partner

e. Any AGYW who believes she is at high risk for HIV infection

f. Any AGYW regardless of risk

\section{Stigma}

1. Providing PrEP endorses the negative (sexual) risk behaviors of users

2. PrEP should not be given to AGYW because they should not be having sex

3. AGYW are not responsible enough to be able to adhere to PrEP

4. AGYW should be ashamed to ask their provider for PrEP 
5. Parents should be ashamed if their young daughters ask their provider for PrEP

\section{Service Delivery Considerations/Service Access and Quality}

1. What do you consider appropriate service settings to deliver PrEP to AGYW? [Check all that apply]

- Public sector health centers

- Private health centers

- Non-governmental organizations

- Faith-based organizations

- Youth-friendly health service locations

- HIV counseling and testing sites

- STI prevention and treatment sites

- Family planning and reproductive health sites

- Maternal health settings

- Gender-based violence counseling sites

- Mobile health services

- Other [please write]

\section{Checklist for service delivery readiness}

Note: The questions below can be thought of as a checklist to determine the readiness of a service delivery site to introduce PrEP for AGYW.

Please respond to the following statements, thinking about your own service setting/facility. Response categories for the following questions are: (a) strongly agree; (b) agree; (c) neutral; (d) disagree; and (e) strongly disagree.

1. Written medical guidelines, protocols and standards for ARV-based prevention, including PrEP, are available at this site

2. Providers have been trained in the provision of PrEP to AGYW

3. Providers have job aids and information, education and communication materials to provide information about PrEP that are appropriate for AGYW

4. Providers counsel clients about HIV testing and repeat testing, HIV prevention options, including male and female condoms, and provide instructions on use, side effects and follow-up required

5. Providers emphasize the importance of condom use as the best available means for HIV prevention and encourage use of male and female condoms along with PrEP

6. Facilities have the necessary equipment and supplies to provide PrEP (including HIV test kits, PrEP, male and female condoms, other) and have a system to prevent stock outs

7. The facility provides a private space for confidential HIV testing and counseling on PrEP use, and providers ensure client privacy and confidentiality

8. Providers tailor interactions to each AGYW's particular needs, circumstances and concerns

9. Providers treat AGYW with dignity and respect; they are non-judgmental and sensitive to gender differences and power imbalances

10. Providers use language that clients, including AGYW, understand, assess knowledge gaps, and encourage questions to check comprehension of information provided 


\section{MALE PARTNERS OF AGYW}

Engaging male partners of AGYW is important in understanding the likelihood of PrEP acceptance, uptake, and adherence by AGYW. Gender norms and the gendered division of power often result in male partners exerting influence over women's decisions about their sexual and reproductive health. Therefore, PrEP introduction efforts need to engage AGYW's male partners to understand the norms and decision-making processes within their relationships, their knowledge and perceptions of PrEP, and their attitudes and concerns toward AGYW's use of PrEP.

\section{Population}

Men's influence on women's decision-making processes regarding their sexual and reproductive health differs by partner type. Research from several microbicide trials found that women reported better adherence and greater ease using products discretely with "casual," transactional, and non-cohabitating partners compared to steady/main partners. ${ }^{25}$ Given the challenges of using HIV prevention interventions in more stable partnerships, we recommend that husbands, cohabitating partners, and boyfriends be the focus of the assessment.

\section{Key Domains}

\section{PrEP Attributes, Knowledge and Perceptions:} One of the reasons why men might be opposed to their partners using sexual and reproductive health products is their concerns about product safety. ${ }^{25}$ Resistance is also fueled by lack of knowledge about the product. Therefore, efforts should be made to elicit their opinions and perceptions regarding PrEP, which can aid in tailoring messages that gain men's support of their partners' use of PrEP. ${ }^{23}$
Gender Norms and Power Dynamics: Research shows that prevailing gender norms about sexuality and relationship dynamics affect how women make decisions about the use of sexual and reproductive health products, including microbicides, condom use, and contraceptive

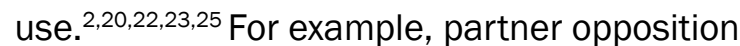
has been identified as an important reason for contraception misuse and that emotional, physical, and sexual violence within relationships significantly reduces contraceptive use and continuation..$^{22,25}$ Furthermore, the choice to use sexual and reproductive health products could have a negative impact on the relationship because some men may equate their usage with infidelity. ${ }^{25}$ Program planners should assess how gender norms and power dynamics influence men's acceptance and support of their partner's use of PrEP.

\section{Methods}

We recommend the use of IDls to gain information on male partners' knowledge, attitudes, beliefs, and concerns about PrEP and their partners' use of it. IDIs are preferred due to the sensitive nature of the questions. A minimum of four to six IDIs should be conducted per relationship type-married, co-habitating, and boyfriend. IDIs should consider the HIV status of the male partner, as HIV positivity may influence men's attitudes toward PrEP. Program planners must also consider regional variations which require a similar number of IDIs for each distinct area.

Recruiting a selection of male partners could be done through their female partners (though there are ethical challenges to consider), through community groups, and through locations where they are known to frequent (e.g., local bars or work venues). One type of location to find AGYW who might have a steady/main partner is health care settings, especially antenatal care and family planning service locations. AGYW might be 
able to directly recruit their partners or they can provide the locations (e.g., home address) where to reach them. It is vitally important that program planners make sure that AGYW are comfortable recruiting their male partners or providing information. Another avenue to recruit male partners is to directly approach them in locations where they are known to frequent. These locations can be identified through their female partners and from key informants, such as staff at NGOs who work with men and are knowledgeable of the local scene. HIV-positive male partners can be recruited through health services, such as those offering services to discordant couples.

Recruit and train male facilitators from the region where the IDIs will take place because they will be knowledgeable and aware of the gender norms and beliefs in that area. Furthermore, being interviewed by peers who are familiar with local norms will build rapport between the facilitator and participants, encouraging honest answers from participants.

\section{Questions}

\section{PrEP attributes, knowledge, and perceptions}

- Had you heard about PrEP before today? If so, what had you heard, and from who or where did you hear it? (Probe for health care provider, friend, community-based educator, PrEP user)

- Based on what you learned today/have heard before, what are your thoughts about PrEP?

- What concerns do you have about PrEP? (probe on safety, stigma, taking a drug if HIV-negative, side effects, relationship concerns, community influences)

- What questions do you have about PrEP?

- Who do you think should have access to this new intervention?
- Which groups would most benefit from PrEP?

- For each group that would benefit, where and how would they best be reached?

- What are the best ways to engage men regarding PrEP?

- What information should be included in education and sensitization campaigns about PrEP geared toward male partners?

-What about you, are you interested in taking PrEP? Why or why not?

- What do you think about AGYW having access to PrEP?

\section{Gender norms and power dynamics}

- Please tell me about instances where you have discussed avoiding/preventing HIV with your primary sexual partner? (Probe on condom use, male circumcision)

- How do you think your opinion affects the decisions your sexual partner makes about using products to prevent HIV and pregnancy, such as condoms or contraceptives?

- How might you discuss your desire to start taking PrEP with her?

- If your sexual partner told you that she wanted to take PrEP, what would be your reaction?

- What would you do?

- What would say? Would you support her

- How would your opinion affect her decision to take PrEP?

- What are the best ways for your partner to engage you about her desire to take PrEP?

- What would be some reasons why your sexual partner would not tell you she was interested in taking PrEP or she was taking PrEP?

- If you found out that your sexual partner was taking PrEP and she had not told you, what would be your reaction?

- What would you do? 
- What would you say?

- If your partner decided to take PrEP, what impact might her decision have on your relationship?

- If your partner wanted to take PrEP, what can be done to gain your support for her decision?

- If your partner decided to take PrEP, what things would you do to support her decision?

- Would you participate in drug-adherence counseling? Why or why not?

- What are the main barriers to you accompanying your partners to the clinic for PrEP? 


\section{PARENTS AND GUARDIANS OF AGYW}

Parents' and guardians' knowledge, attitudes, and perceptions about PrEP may directly influence those of AGYW. This is particularly important to examine in places where the ability to use PrEP by AGYW under the legal age of consent is dependent on obtaining a parent's or guardian's consent. Obtaining parental or guardian consent can be a significant barrier, either because they cannot be reached or because they may decline consent. To determine the feasibility of PrEP for AGYW, especially those who are under the legal age of consent for medical treatments (which varies by country), this section provides guidance on engaging parents and guardians in the assessment process.

\section{Population}

AGYW reside in different types of family structures, where guardians might have varying opinions about their use of medical interventions. Research shows that family structure influences adolescent beliefs as well as health and education outcomes. ${ }^{26,27}$ Understanding how different type of guardians view PrEP for AGYW will aid in tailoring introduction strategies. Examples of family structures to consider appear in Box 4.

\section{Key Domains}

\section{PrEP Attributes, Knowledge and Perceptions:}

Parents and guardians may be reluctant to provide consent for their adolescent to take PrEP because they lack knowledge about it and have safety concerns related to taking the drug. ${ }^{28}$ Without proper information, they might overestimate the risks of adverse health events associated with taking PrEP. Efforts should be made to educate parents and guardians about PrEP and its attributes, especially information about safety,
BOX 4

Potential family structures

Two parent households

Single parent households

Grandparent-headed households

Households headed by other relatives

Household headed by non-relatives

Households with orphans

AGYW living in government facilities

and elicit their opinions regarding PrEP. This information can aid in tailoring messages that foster parents' and guardians' support for PrEP.

Stigma and Social Acceptability: As previously noted, the willingness of AGYW to use and adhere to PrEP will greatly depend on social understandings, including whether it is seen as an effective, healthy, and socially acceptable strategy for HIV prevention among AGYW. ${ }^{21}$ Parents' and guardians' understanding and acceptance of PrEP may play a key role in providing AGYW with support or formal consent to take PrEP, as well as influence AGYW's uptake and adherence to PrEP. However, the stigma associated with taking an ARV medication may result in parents' and guardians' reluctance to provide their consent. Parents may be concerned about the backlash their child and their entire family might face (e.g., belief that their child is HIV-positive) if the community finds out and is not accepting of PrEP. ${ }^{28}$ Introducing PrEP to AGYW, especially those under age 18 (or the legal age of consent), requires understanding the role of stigma and acceptability from the perspective of parents and guardians.

Legal and Ethical Considerations: Many ethical and legal considerations pose a significant 
challenge to providing PrEP to AGYW. The need for adult consent in some settings may act as a deterrent for AGYW in accessing health services due to the need to disclose their behaviors to their parents or guardians. ${ }^{10}$ However, a prevailing question is whether parents and guardians think it is necessary for them to provide consent. Even if parents and guardians think their consent is necessary, there might be circumstances in which their consent can be waived. There is a need to understand these ethical and legal complexities from the viewpoint of parents and guardians if PrEP will be feasible for AGYW under the legal age of consent.

\section{Methods}

Use FGDs to elicit in-depth information on parents' and guardians' knowledge, attitudes, and perceptions about PrEP and considerations around providing consent for their adolescent child to use PrEP. For each chosen family structure type, an ideal focus group size is between 6 and 10 participants, with at least 2-3 groups of participants. Program planners must also consider if groups should be conducted in various regions, which will require a similar number of FGDs (one to two) per chosen area. It is recommended that trained facilitators from the community conduct the FGDs because these individuals will be knowledgeable of local norms and will be able to build rapport with parents.

One avenue for reaching parents is through institutions that service adolescents and/or guardians, such as schools, churches, and social service organizations. For example, teachers in schools can provide information on potential guardians to recruit. Another avenue is to partner with local NGOs who work with families or are familiar with families in their communities. The staff of these organizations can identify the households and aid in recruitment.

\section{Questions}

\section{PrEP attributes, knowledge and perceptions}

- Had you heard about PrEP before today? If so, what had you heard, and who or where did you hear it? (Probe for health care provider, community-based educator, another parent or guardian, PrEP user)

- Based on what you learned today/have heard before, what are your thoughts about PrEP?

- What concerns do you have about PrEP?

- What questions do you have about PrEP?

- Who do you think should have access to this new intervention?

- Which groups would most benefit from PrEP?

- For each group that would benefit, where and how would they best be reached?

- What do you thinking about adolescents and young people having access to this intervention? Should PrEP be a choice for youth?

- What about girls?

- At what stage in life do you think girls should be able to access PrEP?

- What about boys?

- Have you ever discussed HIV prevention with your sons or daughters?

- If your daughter wanted to take PrEP or you found out that your daughter was taking PrEP, what would be your reaction to this news? Why would this be your reaction?

\section{Stigma and social acceptability}

- Who do you consider to be the most at risk for HIV? Why?

- What about AGYW and sub-groups (List the various sub-groups of AGYW if not discussed) 
- What do you think parents like yourself think about adolescent girls who engage in sexual activity?

- Do you think AGYW are able to assess their HIV risk?

- How do other adults in the community, who are like you, view AGYW who take ARVs currently?

- How would you think they would view those who take PrEP?

- How do you think they would view you if they knew your child was taking PrEP?

- Sometimes, people who are taking ARVs face negative attitudes or reactions from health care providers or from other community members; what drives these attitudes or reactions?

- What might be the best way of addressing these issues to reduce negative attitudes and reactions?

- If your daughter wanted to take PrEP or you found out that your daughter was taking PrEP, what would be your reaction to this news? Why would this be your reaction?

- If your daughter wanted to take PrEP or you found out that your daughter was taking PrEP, what kind of support would you offer to her?

- Would you be willing to attend adherence counseling with your adolescent? Why or why not?

- What should be done to inform parents about PrEP? What will gain your support if you are reluctant about your daughter taking PrEP?

- What information should be included in education and sensitization campaigns about PrEP geared toward parents?

- What cultural traditions might impact AGYW decision to use PrEP? How about to not use PrEP?

\section{Legal and ethical considerations}

Note: These questions should be tailored based on the legal and ethical guidelines within each country.

- Currently, parents/guardians must give adolescents under the age of [insert relevant age] their permission to access [insert relevant services] and use [insert relevant products]. Under what circumstances do you think parents in your community would give their adolescents permission to access these services or use these products?

- What has been your other parents' experience with offering permission for services?

- Currently, parents/guardians must give adolescents under [insert relevant age] their permission to access [insert relevant services] and use [insert relevant products]. If policy makers decide that adolescents no longer require parental permission to access these services or use these products, what would be your reaction? Why would you have such a reaction?

- What about PrEP, do you think parental permission should be required in order for adolescents to use PrEP? Why or why not?

- Under what circumstances do you think that your adolescent does not need your permission to access [insert relevant services] and use [insert relevant products]? What about PrEP, specifically?

- If your daughter wanted to take PrEP, would you be willing to give your permission for her to take it?

- What are the main reasons why you would not give your consent?

- What are the main reasons why you would give your consent? 


\section{POLICY MAKERS}

Understanding policy makers' awareness, attitudes, perceptions, and preferences around PrEP is very important in guiding its introduction and roll-out. It is also important to understand their vision for how PrEP fits into the overall HIV prevention landscape in their countries and their opinions about drug availability, prioritization of populations, settings for PrEP roll-out, tailoring services to foster effective uptake of PrEP, and ensuring adherence. ${ }^{4}$

To engage policy makers around PREP and AGYW, we recommend the toolkit, "Introducing antiretroviral (ARV)-based prevention products for women: a guide to strategic decision-mak- ing and planning." With the support of USAID/ Washington, the Population Council developed the toolkit (http://www.popcouncil.org/uploads/ pdfs/2015HIV_ARVtoolkit.pdf) for use by governments, organizations, and donors that are in a position to support or add new HIV prevention products into existing programs. This toolkit is based on the belief that women should have access to a wide range of options to prevent HIV and have the ability to choose those that best meet their needs. The toolkit was piloted in Zimbabwe and a document containing the results along with additional resources can be downloaded at http://www.popcouncil.org/uploads/ pdfs/2015HIV_ZimbabweProviderWorkshop.pdf. Box 5 summarizes three of the toolkit's modules.

\section{BOX 5 \\ Toolkit modules}

\section{Module 1. Landscape analysis}

An analysis of the epidemiologic context of the HIV epidemic, along with an HIV policy and program scan. The analysis consists primarily of a desk review of available literature and data, supplemented as needed with input from experts familiar with the HIV epidemic and the specific country context. It serves as a starting point and foundation for the process in Modules 2 and 3 .

\section{Module 2. Discussion guide for key opinion leaders and decisionmakers}

A guide to conducting IDIs with a select group of key decision makers who will be involved in or influence strategic decisions about the introduction and roll-out of PrEP. Key domains to cover with opinion leaders and policy makers include: scientific evidence and data needs; target population and priorities; impact on health systems; policy considerations; economics, cost, and financing; and decision-making. IDIs should be conducted by a senior leader who is knowledgeable about both HIV prevention and the country's policy environment. Results will provide an indication of the strategic role PrEP could play within a country's HIV prevention program and help determine how best to continue to explore and plan for introduction.

\section{Module 3. Program planning guide}

The guide is to be used with policy makers, service providers, and program managers who are responsible for implementing HIV prevention programs. The module guides workshop participants to consider the feasibility of introducing HIV prevention tools in specific facility-based settings by guiding them through a series of design considerations to determine opportunities and challenges in service setting(s) that should be addressed in program planning. 


\section{SECTION 3 ADDITIONAL DATA COLLECTION METHODS}

In this document we propose the use of FGDs and IDIs. FGDs involve a small group of individuals who share common characteristics or experiences related to the topic of interest. ${ }^{33}$ The group dynamic allows for brainstorming to generate insights and strategies, provides information on social context such as norms, and gain insights into social interactions. IDIs, on the other hand, are one-on-one discussions between a participant and an interviewer. ${ }^{34}$ The interview can be semi-structured with open-ended questions or unstructured allowing the participant to freely discuss the topic. IDIs provide rich, detailed information from participants about their experiences, opinions, and behaviors. They are particularly useful for sensitive and complex subjects. Key informant interviews are IDIs with people who are knowledgeable about what is going the community.

\section{PARTICIPATORY TECHNIQUES}

As part of FGDs and IDIs, there are additional techniques that can be used to get more insight into participants' views:

1. Card-sorting involves creating a set of cards with descriptions about a topic, problem, behavior, or product. The descriptions can be pictorial or text. The cards are given to the participant who is asked to sort them in a particular order. For example, the researcher might ask AGYW to sort cards on PrEP characteristics and attributes according to what most concerns them about PrEP or what would most influence their decision to take PrEP. After the sorting is complete, the researcher can ask participants questions to further explain their thought process for the order they selected. Card-sorting can be incorporated into qualitative study designs previously discussed. For example, participants can complete a card-sorting activity at the end of the FGD.

2. Vignettes are stories created by researchers about individuals and situations to help elucidate participants' perceptions, beliefs and attitudes about a topic under study. ${ }^{35,36}$ The premise of the stories comes from a range of sources, including previous research findings, research participants, and information from key informants in the field such as NGO staff working with the research population. It is important that the vignettes are relevant and real to the participants. The vignettes are presented to participants, who are asked to respond to a range of questions about what they have heard, including what they would do in a particular situation or how they think another person would respond. It is particularly useful when interviewing young children and adolescents. One of the key limitations is that vignettes cannot account for what a 
participant will actually do in real life but it is helpful for unpacking perceptions, beliefs, and attitudes on a wide range of social issues.

\section{OBSERVATIONS}

Observation involves the careful and systematic documentation of events and behaviors in the social setting chosen for study. The observer makes detailed field notes that are nonjudgmental and concrete in descriptions. ${ }^{37,38}$ Notes should include descriptions of actions and interactions among individuals as well as non-verbal cues such as body language and facial expressions. After initial observations, checklists can be created to assess the presence or absence of a phenomenon. One limitation to this method is reactivity by those being observed; individuals might change their behaviors when they know they are being observed. One solution is to visit the site for a few days before data collection to reduce reactivity.

Program planners can directly observe the clinical settings in which PrEP is likely to be offered as well as current interactions between providers and AGYW patients to understand service delivery areas that work well and areas requiring improvement. For example, observing interactions surrounding contraceptive use can serve as a proxy for PrEP. This information can be used to prepare a clinical setting for PrEP introduction, such as trainings of health care providers. Following the introduction of PrEP, observations can be conducted to understand how well the setting was prepared, successful implementation strategies, and ongoing challenges. order to evaluate client experience when seeking services, such as health care. ${ }^{39-41}$ The method reduces the bias that can be introduced in observations; that is, where individuals change their behaviors because they know they are being observed. Mystery clients are individuals who possess the characteristics of the types of patients who would seek the service. Clients are trained to assume a specific role when seeking services and then report on their experience. Immediately following the visit, the client is interviewed using a checklist or interview guide in order to capture a detailed account about their experiences and interactions while in the facility. It is best that mystery clients do not come from the area where the clinical setting is located. The facility should be informed that mystery clients will visit the facility but not exact dates. For example, facilities can be told mystery clients will visit within the next three months.

Program planners can use mystery clients during PrEP introduction to monitor and evaluate service delivery, including patient-provider interactions, adherence to delivery protocols, and service quality. This method can provide detailed and useful insights into good practices and areas for improvement.

\section{MYSTERY CLIENT METHOD}

The mystery client (or shopper) method involves researchers posing as prospective clients in 


\section{SECTION 4}

\section{DATA ANALYSIS STRATEGIES}

\section{QUALITATIVE DATA}

Analysis and interpretation of the qualitative and narrative data proposed in this guidance document should provide unique insights that can assist in determining the feasibility of introducing PrEP to AGYW and if feasible, the development, design, and implementation of PrEP introduction strategies. This section is not intended to provide a comprehensive data analysis plan because there are several good textbooks that can provide guidance on approaches to qualitative data analysis. ${ }^{29-39}$ This section, instead, provides practical advice for conducting qualitative analyses.

Key strategies for analyzing qualitative data include designing an analysis plan, becoming familiar with the data, categorizing the data, identifying patterns and connections within the data, and interpreting the data. There are several qualitative data analysis software programs that can aid in the analysis, including ATLAS.ti, NVivo, MAXQDA, Dedoose, and QDA Miner. All of these programs have strengths and weaknesses and choosing one will depend on the types on analyses that will be performed. Each program has user-friendly resources to aid in conducting the analyses.

Recommended strategies for conducting qualitative data analyses:

- Design an analysis plan: A data analysis plan helps in structuring the analyses and ensuring all team members are informed of how the data will be analyzed. Design an analysis plan that reflects the aims of the data collection and the questions that program planners are trying to answer. Program planners might want to focus the analyses by topic, an event, or learnings from a group.

- Outline transcription expectations: Data collected are usually transcribed into written text for analyses. Good quality transcription is fundamental to the data analysis process. The level and detail of transcription will vary and it is important to outline these expectations from the beginning. For example, decisions must be made regarding whether verbal and non-verbal information should be included. The decisions on transcription are dependent on the research aims.

- Start the analysis as data is being collected: Analyses of qualitative data is an iterative process that starts during data collection and continues beyond data collection. As data is collected, program planners should listen to recordings and read transcripts on an ongoing basis in order to understand the quality of the data and to refine questions and explore emergent topics not previously considered in subsequent interviews or group discussions. Additionally, field notes should include a summary of the interview and these notes should be reviewed continually.

- Coding and analysis: The next step in the analysis process usually involves data condensation or reduction process, through coding the data and drafting analytic memos, pertaining to the major themes emergent from the data and/or the themes of interest. It is 
important at this stage of the analysis process to develop a codebook, particularly if multiple research team members will be involved in reviewing and coding the data. The codebook aids in the systematic coding of text. For each code (theme), the codebook outlines the name of the code, its definition, guidelines for when to use and not to use the code, and examples. Due to the iterative nature of qualitative data analysis, the codebook can be refined several times during the analysis.

- Data display: Data display (through matrices, flow charts, etc.) can assist in assembling data across themes and respondents to better elucidate the relationships among themes, groups of respondents, and categories of data. This critical step allows for comparison and contrasting of findings across and within respondents.

- Assess reliability and validity: It is important to assess the validity and reliability of findings to ensure that the findings reflect the reality of the participants' lives and that the findings can be consistent, respectively. Closely rereading transcriptions, triangulating data from various sources, and obtaining feedback from participants are common methods for assessing reliability and validity.

- Interpretation of findings: One of the most important steps in qualitative analysis involves the interpretation of findings, drawing conclusions, and verification of the data interpretation. In this final stage, it is important to contextualize the findings with existing literature, connect to theory, and discuss alternate explanations/interpretations of the data.

\section{SURVEYS}

Surveys are a method of gathering information from a defined group of people to get a broader understanding of the subject. Surveys mostly consist of close-ended questions. Traditionally, surveys have been used to generalize to the larger population. Surveys allow for large samples and standardized responses. Technology, when used, increases the ease of survey administration, allowing for quick collection of data and minimization of errors. Care is needed when designing surveys and analyzing their results, to ensure that the right questions are being asked, the right people are taking the survey, the appropriate number of people of surveyed, and the results are valid.

Several courses and textbooks can provide specific quidance on quantitative survey data analysis. $^{42}$

\section{Conjoint Analysis}

Conjoint analysis, a development of market research, is a set of techniques used to study customers' decision-making processes and determining tradeoffs. ${ }^{43-45}$ Conjoint analysis has the ability to answer various "what if" questions based on the results of an analysis of data collected on hypothetical and real choice alternatives. The method uses a survey that outlines the attributes and characteristics about a product, which is administered to a sample of the population that the product will eventually target. Various analytic models, with the assistance of easy-to-use software, are conducted using the collected survey data to predict how users will choose products and services.

Conjoint analysis can be used to elicit potential PrEP users' views of the drug, including the importance and trade-offs of different characteristics (e.g., frequency of use, HIV testing requirement) of the drug. Additional factors that can be assessed in conjoint analyses include identifying priority healthcare settings for providing PrEP as well as the relative importance of different contextual influences (e.g., partner preferences, stigma) on product views. 


\section{SECTION 5 RESEARCH UTILIZATION}

Research utilization is the process by which research findings are synthesized and disseminated to make an impact on or change in existing practices. Translating findings into action at the national, sub-national, and service delivery levels requires two-way information exchange between the people who can apply research findings (i.e., stakeholders) and researchers or program planners.

Research utilization is a critical component of the research process and a necessary step for developing PrEP introduction strategies for AGYW. In Box 6 (page 34), we highlight seven practices that increase the likelihood that key stakeholders will promote and utilize study findings and recommendations to make programmatic and policy decisions. These practices are drawn from a guidance document developed by Project SOAR (Supporting Operational AIDS Research), an operations research project led by the Population Council. This document can guide the planning and implementation for effective research utilization. We recommend program planners and researchers access the complete resource, "Project SOAR's research utilization process: guidance document and toolkit," at http://www.popcouncil. org/uploads/pdfs/2016HIV_ProjectSOAR_ResUtilization.pdf. 


\section{BOX 6}

\section{Research utilization practices}

Practice 1: Identify key stakeholders as early as possible to understand their priority information needs and establish the audience(s) for the findings. This will enhance the study's relevance among those implementing HIV programs and services and development partners investing in the programs.

Practice 2: Engage stakeholders during study design to ensure that the methods, intervention (if there is one), and data collection processes reflect and respond to the priorities and needs of the HIV program and services.

Practice 3: Establish a study-specific advisory panel that assigns roles and responsibilities to key stakeholders. This will help ensure that stakeholders remain engaged throughout the study process and will want to champion the use of findings once they become available.

Practice 4: Engage stakeholders during data collection to reinforce their involvement and deepen their understanding of the research process and what the study is investigating. This can also help improve fidelity to the intervention under study, if there is one. Engaging key stakeholders during data collection may motivate them to later use findings and disseminate them among a wider audience.

Practice 5: Work together with stakeholders to interpret findings and develop recommendations. Engaging stakeholders permits them to review preliminary analyses and offer insight into the local program context, which informs specific and realistic recommendations.

Practice 6: Produce the study report and hold the dissemination meeting, during which stakeholders finalize a plan to use findings and recommendations.

Practice 7: Coordinate the implementation of the plan to use findings and recommendations. 


\section{REFERENCES}

1. Joint United Nations Programme on HIV/AIDS. 2014. "The gap report." Geneva: UNAIDS.

2. Celum, CL et al. 2015. "Rethinking HIV prevention to prepare for oral PrEP implementation for young African women," Journal of the International AIDS Society $18(4$ Suppl 3): 20227.

3. Fonner, VA et al. 2016. "Effectiveness and safety of oral HIV pre-exposure prophylaxis (PrEP) for all populations: A systematic review and metaanalysis," AIDS, published online ahead of print.

4. World Health Organization. 2015. "Guideline on when to start antiretroviral therapy and on preexposure prophylaxis for HIV." Geneva: World Health Organization.

5. Baeten, JM et al. 2012. "Antiretroviral prophylaxis for HIV prevention in heterosexual men and women," New England Journal of Medicine 367(5): 399-410.

6. Van Damme, L. et al. 2012. "Preexposure prophylaxis for HIV infection among African women," New England Journal of Medicine. 367(5): 411-422.

7. Corneli, AL et al. 2014. "FEM-PrEP: adherence patterns and factors associated with adherence to a daily oral study product for pre-exposure prophylaxis," Journal of Acquired Immune Deficiency Syndromes. 66(3): 324-331.

8. Henderson, $\mathrm{F}$ et al. "Characteristics and oral PrEP adherence in the TDF2 open-label extension in Botswana." Paper presented at 8th IAS Conference on HIV Pathogenesis Treatment and Prevention, Vancouver, Canada, 18-22 July.

9. PEPFAR. 2016. Working together for an AIDS-free future for girls and women. http://www.pepfar. gov/partnerships/ppp/dreams/, Washington, DC: Department of State.

10. UNICEF. 2015. Consultation on Clinical, Ethical and Operational Considerations for the implementation of Oral PreExposure Prophylaxis (PrEP) in Sexually Active Older Adolescents
(15-19) at High Risk of HIV Infection: Meeting Report. New York: UNICEF.

11. Brady, M and E McGrory. 2012. "Shaping the operations research agenda for antiretroviralbased prevention products for women: gels and rings: consultation report." New York: Population Council.

12. Population Council. 2015. "Introducing antiretroviral (ARV)-based prevention products for women: a guide to strategic decisionmaking and planning." New York: Population Council.

13. Underhill, $K$ et al. 2010. "Implementation science of pre-exposure prophylaxis: preparing for public use," Current HIV/AIDS Reports 7(4): 210-219.

14. Underhill, $\mathrm{K}$ et al. 2010. "Packaging PrEP to prevent HIV: an integrated framework to plan for pre-exposure prophylaxis implementation in clinical practice," Journal of Acquired Immune Deficiency Syndromes (1999). 55(1): 8.

15. Mensch, BS, A Van Der Straten, and LL Katzen. 2012. "Acceptability in microbicide and PrEP trials: current status and a reconceptualization," Current Opinion in HIV and AIDS 7(6): 534.

16. McLeroy, KR et al. 1988. "An ecological perspective on health promotion programs," Health Education \& Behavior 15(4): 351-377.

17. World Health Organization. 2014. Health for the World's Adolescents: A second chance in the second decade. Geneva: World Health Organization.

18. Baral, S et al. 2012. "Burden of HIV among female sex workers in low-income and middleincome countries: a systematic review and metaanalysis," The Lancet Infectious Diseases 12(7): 538-549.

19. Corneli, A et al. 2015. "Preparing for the rollout of pre-exposure prophylaxis (PrEP): a vignette survey to identify intended sexual behaviors among women in Kenya and South Africa if using PrEP," PLOS ONE 10(6): e0129177. 
20. Carroll, JJ et al. 2016. "Gendered differences in the perceived risks and benefits of oral PrEP among HIV-serodiscordant couples in Kenya," AIDS Care. 11 Jan, online ahead of print.

21. Haire, BG. 2015. "Preexposure prophylaxisrelated stigma: strategies to improve uptake and adherence-a narrative review," HIV/AIDS (Auckland, NZ). 7: 241.

22. Delany-Moretlwe, $\mathrm{S}$ et al. 2016. “Planning for HIV preexposure prophylaxis introduction: lessons learned from contraception," Current Opinion in HIV and AIDS. 11(1): 87-93.

23. Woodsong, $C$ et al. 2013. "Microbicide clinical trial adherence: insights for introduction," Journal of the International AIDS Society 16(1): 18505.

24. Edelman, EJ, D Smith, and DA Fiellin. 2013. "Moving HIV pre-exposure prophylaxis into clinical settings: lessons from buprenorphine," American Journal of Preventive Medicine 44(102): S86.

25. Lanham, $M$ et al. 2014. "Engaging male partners in women's microbicide use: evidence from clinical trials and implications for future research and microbicide introduction," Journal of the International AIDS Society 17(3 Suppl 2): 19159.

26. Mensch BS, Bruce J, Greene ME. 1998. The Uncharted Passage: Girls' adolescence in the developing world. New York: Population Council. 1998.

27. Pilgrim, NA et al. 2013. "Sexual coercion among adolescent women in Rakai, Uganda: does family structure matter?," Journal of Interpersonal Violence 28(6): 1289-313.

28. DiClemente, RJ, JM Sales, and N Borek. 2010. "Barriers to adolescents' participation in HIV biomedical prevention research," Journal of Acquired Immune Deficiency Syndromes (1999) 54(Suppl 1): S12.

29. Miles, M, A Huberman, and J Saldaña. 2014. Qualitative Data Analysis. A Methods Sourcebook. Thousand Oaks, CA, Sage Publications.

30. Guest, G, KM MacQueen, and EE Namey. 2011. Applied thematic analysis. Thousand Oaks, CA, Sage Publications.

31. Saldaña, J. 2015. The Coding Manual for Qualitative Researchers. Thousand Oaks, CA, Sage Publications.
32. Miles, M and AM Huberman. 1994. Qualitative Data Analysis: An Expanded Sourcebook. Thousand Oaks, California: Sage.

33. Barbour R. 2008. Doing Focus Groups. Thousand Oaks, California: Sage.

34. Kvale S. 2008. Doing interviews. Thousand Oaks, California: Sage.

35. Barter, C and E Renold. 2000. "I wanna tell you a story': exploring the application of vignettes in qualitative research with children and young people," International Journal of Social Research Methodology 3(4): 307-323.

36. Hughes, R. 1998. "Considering the vignette technique and its application to a study of drug injecting and HIV risk and safer behaviour," Sociology of Health \& IIIness 20(3): 381-400.

37. Marshall, C and GB Rossman. 2014. Designing Qualitative Research. Thousand Oaks, CA, Sage Publications.

38. Patton, MQ. 2005. Qualitative Research. Wiley Online Library.

39. Jacob, S, N Schiffino, and B Biard. 2016. "The mystery shopper: a tool to measure public service delivery?," International Review of Administrative Sciences 14 Mar, online ahead of print.

40. Rhodes, K. 2011. "Taking the mystery out of 'mystery shopper' studies," New England Journal of Medicine 365(6): 484-486.

41. Bauermeister, JA et al. 2015. "The use of mystery shopping for quality assurance evaluations of HIV/STI testing sites offering services to young gay and bisexual men. AIDS and Behavior 19(10): 1919-1927.

42. Aday, L. and L Cornelius. 2006. Designing and Conducting Health Surveys: A Comprehensive Guide. San Francisco: Jossey-Bass.

43. Rao, VR. 2014. Applied Conjoint Analysis. New York/London: Springer-Verlag Berlin Heidelberg.

44. Gustafsson, A, A Herrmann, and F Huber. 2013. Conjoint Measurement: Methods and Applications. New York/London: Springer-Verlag Berlin Heidelberg.

45. Ryan, M and S Farrar. 2000. "Using conjoint analysis to elicit preferences for health care," British Medical Journal 320(7248): 1530. 

Population Council 4301 Connecticut Ave, NW,

Suite 280

Washington, DC 20008

USA

popcouncil.org 